\title{
Neutron asymptotic normalization coefficients and halo radii of the first excited states of ${ }^{13} \mathrm{C}$ and ${ }^{11} \mathrm{Be}$
}

\author{
T. L. Belyaeva ${ }^{1 \mathrm{a}}$, R. Perez-Torres ${ }^{2}$, A. S. Demyanova ${ }^{3}$, S. A. Goncharov ${ }^{4}$, and A. A. Ogloblin ${ }^{3}$ \\ ${ }^{1}$ Universidad Autónoma del Estado de México, C. P. 50000, Toluca, Mexico \\ ${ }^{2}$ Instituto Tecnológico de Toluca, Metepec, Estado de México, C. P. 52140, Mexico \\ ${ }^{3}$ RRC Kurchatov Institute, Moscow RU-123182, Russia \\ ${ }^{4}$ Lomonosov Moscow State University, Moscow 119901, Russia
}

\begin{abstract}
We have performed coupled reaction channels calculations of the (d,p) reactions on ${ }^{12} \mathrm{C}$ and ${ }^{10} \mathrm{Be}$ at laboratory energies of 12,25 , and $30 \mathrm{MeV}$ leading to the ground and first excited states of ${ }^{13} \mathrm{C}$ and ${ }^{11} \mathrm{Be}$. We found spectroscopic factors $S_{\text {exp }}$, asymptotic normalization coefficients (ANCs) and root mean square radii of the last neutron in these states. Our calculations confirm the existence of neutron halos in the first excited state of ${ }^{13} \mathrm{C}$, as well as in the ground and the first excited states of ${ }^{11} \mathrm{Be}$. We found that the neutron transfer dominates at energies about 12 and $25 \mathrm{MeV}$ and demonstrated that the states with enlarged radii are formed in the reactions of a peripheral type, which satisfy the criterion of a peripherality: $C^{2}=S_{\exp } b^{2}=$ const, where $C$ is the ANC and $b$ is the single-particle ANC.
\end{abstract}

\section{Introduction}

Neutron halos have been almost exclusively observed in ground states (g.s.) of some neutron-rich radioactive nuclei located close to the neutron drip line. Nevertheless, ideas about universality of the halo phenomenon were expressed soon after its discovery (see, e.g., [1]). It is well known that search for halos in the excited states by the direct measurement of the radii of short-living nuclear states located near and above the neutron emission thresholds is complicated due to their very short half-life times $\left(\tau_{1 / 2} \leq 10^{-10} \mathrm{~s}\right)$. Thus, alternative indirect methods are required. Liu et al. [2] reported the observation of halos in the first excited states of ${ }^{13} \mathrm{C}$ and ${ }^{12} \mathrm{~B}$ and analyzed them within the asymptotic normalization coefficient (ANC) method by estimating the radii of these states. The ANC theory for peripheral reactions has been developed over 35 years ago and summarized in the review of Blokhintsev et al. [3]. The modified diffraction model (MDM) is the other method developed recently [4-6] for determining the nuclear radii of the short-lived excited states. The MDM was used for determining the radii of the famous Hoyle state $\left(0^{+}, E_{\mathrm{x}}=7.65 \mathrm{MeV}\right)$ and the excited $\left(2^{+}, E_{\mathrm{x}}=9.84\right.$ $\mathrm{MeV}$ ) Hoyle state of ${ }^{12} \mathrm{C}$, and the cluster states of ${ }^{13} \mathrm{C}$ and ${ }^{11} \mathrm{~B}$, differing from the Hoyle state by an extra neutron and a proton hole [4-6]. The MDM was also used to reveal some signatures of neutron halos in the excited states of light nuclei located close to the neutron emission thresholds [7]. In particular, the abnormally large rms radius $(2.74 \pm 0.06 \mathrm{fm})$ was identified for the $3.089-\mathrm{MeV} 1 / 2^{+}$state

\footnotetext{
${ }^{\text {a }}$ Corresponding author: tbl@uaemex.mx
}

This is an Open Access article distributed under the terms of the Creative Commons Attribution License 2.0, which permits unrestricted use, distribution, and reproduction in any medium, provided the original work is properly cited. 
of ${ }^{13} \mathrm{C}$. The radius of a one-neutron halo in this state was estimated to be $5.88 \pm 0.39 \mathrm{fm}$, which is comparable with the calculations of Ref. [2], $5.04 \pm 0.75 \mathrm{fm}$.

In this article, we apply the ANC method to analyse the ${ }^{12} \mathrm{C}(\mathrm{d}, \mathrm{p}){ }^{13} \mathrm{C}$ (g.s., $\left.1 / 2_{1}{ }^{+}\right)$and ${ }^{10} \mathrm{Be}(\mathrm{d}, \mathrm{p})$

${ }^{11} \mathrm{Be}$ (g.s., $1 / 2_{1}{ }^{-}$) reactions at incident deuteron energies $11.8,25.9$, and $30 \mathrm{MeV}$ for the first system, and 12.0 and $25.0 \mathrm{MeV}$ for the second one. We compare our calculations with the results of Refs. $[2,7]$. In spite of the existing great amount of $(d, p)$ and $(p, d)$ data related to the g.s. of these nuclei, there exist only a few data of $(\mathrm{d}, \mathrm{p})$ reaction on ${ }^{12} \mathrm{C}$ and ${ }^{10} \mathrm{Be}$ concerning the excited states of ${ }^{13} \mathrm{C}$ and ${ }^{11} \mathrm{Be}$. We carry out coupled reaction channels (CRC) calculations of the direct proton transfer for these systems and compare the results with the differential cross sections measured in Refs. [2,9-11]. The first aim of our analysis is to extract the experimental spectroscopic factors (SF) $S_{\text {exp }}$ and ANC $C_{\mathrm{A}-n}$ for the A- $n$ systems that form the finite nuclei $\mathrm{B}=\mathrm{A}+n$ both in the g.s. and the excited states. We fit the experimental cross sections at all studied energies applying reasonable optical potentials for the entrance and exit channels, and varying the A- $n$ overlap wave functions. The second aim is calculation of the rms radius of the last neutron in the certain state of the finite nucleus by using obtained values of SFs and ANCs. In the case of a nuclear state possessing a neutron halo, this extracted radius is associated with the rms radius of the neutron halo in this state.

\section{Results and discussion}

Many transfer reactions are actually sensitive only to the tail of the overlap wave functions, i.e., they are peripheral. Numerous studies (see, e.g., Refs. [3, 12]) showed that the model-independent ANC $C_{c v, l j}$ (for the vertex $p \rightarrow c+v$ with the relative orbital angular moment $l$ and the total angular moment $j$ ) is related to the product of the "single-particle" (sp) ANC $b_{c v, l j}$ (which is determined, generally, in the single-particle approximation) and a root square of the spectroscopic factor: $C_{c v, l j}=b_{c v, l j} S_{c v, l j}{ }^{1 / 2}$.

Both latter quantities, as it is well known, are model dependent and can be found from DWBA, CDCC, or CRC analysis of the experimental data. In fact the empirical values of SF, $S_{\exp }(p \rightarrow c+v)$, are determined by a normalization of the calculated cross section to the experimental data. For the peripheral direct nuclear reactions, the combination $C_{c v, l j}^{\exp } \approx b_{c v, l j} S_{\exp }^{1 / 2}$ is almost constant or only weakly depends on model parameters, contrary to the behaviour of empirical values of $S_{\exp }$ and $b_{c v, l}$, separately. We shell call the ANC determined by means of this procedure, the experimental ANC, $C^{\exp }{ }_{c v, l j}$. The persistence of $C^{\exp }{ }_{c v, l j}$ in a number of cross-section calculations with different sp wave functions will be considered as a criterion of the peripherality of this reaction. The distance between core and valence neutron is defined by the rms radius of the overlap wave function $\left|\phi_{A n}(r)\right|$ :

$$
\left\langle r^{2}\right\rangle^{1 / 2}=\left[\int_{0}^{\infty} r^{2}\left|\phi_{A n}(r)\right|^{2} r^{2} d r\right]^{1 / 2} /\left[\int_{0}^{\infty}\left|\phi_{A n}(r)^{2} r^{2} d r\right|\right]^{1 / 2}=S^{-1 / 2}\left[\int_{0}^{\infty} r^{4}\left|\phi_{A n}(r)\right|^{2} d r\right]^{1 / 2}
$$

By using the asymptotic expression (the Whittaker function $W(2 k r)$ ) of the overlap wave function and its single-particle approximation (the sp overlap wave function $u_{A n}(r)$ ), Eq. (1) is changed

$$
\left\langle r^{2}\right\rangle^{1 / 2} \approx\left[\int_{0}^{R_{N}} r^{4}\left|u_{A n}(r)\right|^{2} d r+C^{2} / S_{\exp } \int_{R_{N}}^{\infty} r^{2} W^{2}(2 k r) d r\right]^{1 / 2} .
$$

Here $R_{\mathrm{N}}$ is the nuclear interaction radius. Our theoretical analysis within the CRC model is made using the computer code FRESCO [13]. The full complex remnant term (an additional optical potential (OP) $U_{\mathrm{p}-13 \mathrm{C}}$ calculated using the global parameterization [14]) and the non-orthogonality correction are taken into account. The ${ }^{13} \mathrm{C}$ nucleus in the $1 / 2^{-}$g.s. conforms to the occupation of the $1 p_{1 / 2}$ shell-model orbital, and its first $1 / 2^{+}$excited state at $E_{x}=3.089 \mathrm{MeV}$ located $1.86 \mathrm{MeV}$ below the ${ }^{12} \mathrm{C}+n$ threshold, is associated with the $2 \mathrm{~s}_{1 / 2}$ shell-model orbital. In our calculations, the elastic scattering is coupled with the stripping channel, as well as the inelastic scattering to the first $2^{+}$state of ${ }^{12} \mathrm{C}$. The adopted from Refs. [15, 16] and slightly changed $d+{ }^{12} \mathrm{C}$ OPs at 11.8 and $25.9 \mathrm{MeV}$ reproduced very well elastic data. That is why we do not consider a possibility of deuteron breakup, which, in generally, affects the elastic channel, but its influence is more significant for the heavier targets and higher incident energies [17]. The OPs describing the $p+{ }^{13} \mathrm{C}$ interaction in the exit channel and $p+{ }^{12} \mathrm{C}$ 
interaction at half the incident deuteron energy are calculated using the global parameterization [14] and the interpolation of the OP parameters obtained in Ref. [18]. Calculated average values of the

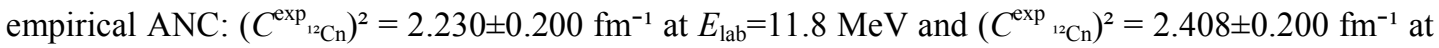
$E_{\mathrm{lab}}=25.9 \mathrm{MeV}$ are in perfect agreement with the corresponding value, $2.46 \pm 0.18 \mathrm{fm}^{-1}$, extracted in Ref. [19] from the DWBA calculations of this reaction. The ${ }^{12} \mathrm{C}\left(\mathrm{d}, \mathrm{p}_{1}\right){ }^{13} \mathrm{C}$ reaction at $E_{\mathrm{lab}}=11.8$ and 30 $\mathrm{MeV}$ leading to the first excited $3.09 \mathrm{MeV} 1 / 2^{+}$state in ${ }^{13} \mathrm{C}$ (see Fig. 1, left panel) exhibits all features of a peripheral process, as the ANC values at both energies remain almost constant under moderate changes of the sp potential parameters, i.e., the peripherality condition is well fulfilled. The ANC values extracted from the $\left(\mathrm{d}, \mathrm{p}_{1}\right)$ reaction, $\left(C^{\exp }{ }^{12} \mathrm{Cn}\right)^{2}=3.034 \pm 0.025 \mathrm{fm}^{-1}$ at $E_{\mathrm{lab}}=11.8$ and $3.456 \pm 0.126$ $\mathrm{fm}^{-1}$ at $E_{\mathrm{lab}}=30 \mathrm{MeV}$ agree very well with those reported by Liu et al. [2], 3.39 $\pm 0.59 \mathrm{fm}^{-1}$. The main result is that the average rms radius of the last neutron in the first excited $3.09 \mathrm{MeV} 1 / 2{ }_{1}{ }^{+}$state of ${ }^{13} \mathrm{C}$ is a factor of 1.75 larger than the rms radius of the last neutron in the $1 / 2^{-}$g.s. of ${ }^{13} \mathrm{C}$. The asymptotic parts of the rms radius contribute as 0.91:0.37, correspondingly. Thus, we can associate the rms radius of the last neutron in the first excited $3.09 \mathrm{MeV} 1 / 2_{1}{ }^{+}$state of ${ }^{13} \mathrm{C}$ with the halo radius, which is found to be equal $R_{\mathrm{h}}\left({ }^{13} \mathrm{C}\right)=5.615 \pm 0.158$. Note that this value is essentially the same for both energies used in the calculations: 11.8 and $30 \mathrm{MeV}$, and perfectly agrees with that determined by the MDM [7].
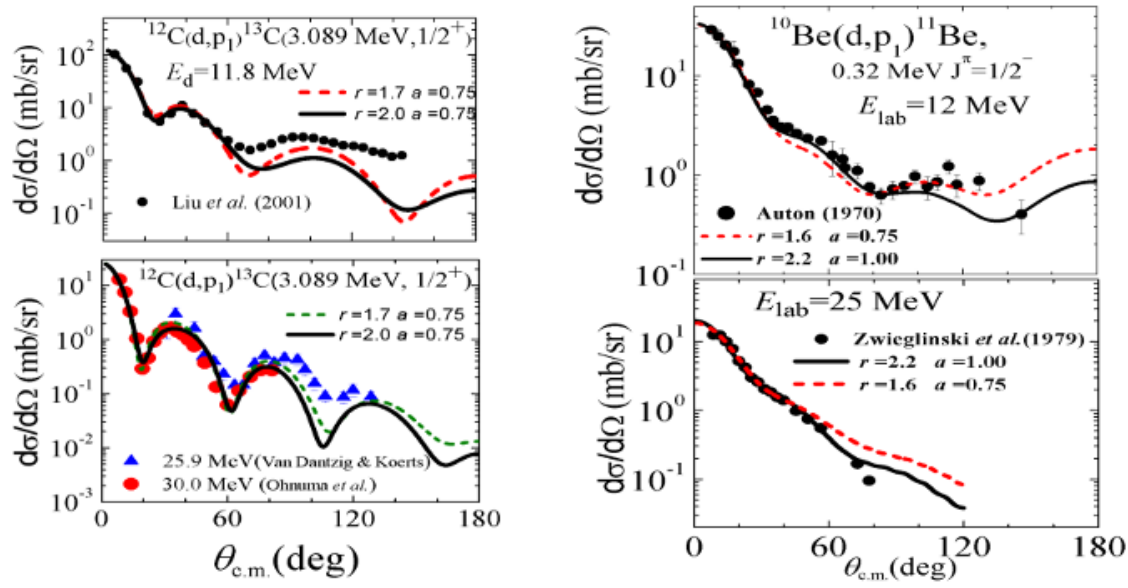

Figure 1. Left panel: $\mathrm{CRC}$ fit of the ${ }^{12} \mathrm{C}\left(\mathrm{d}, \mathrm{p}_{1}\right)^{13} \mathrm{C}$ reaction cross sections at $E_{\mathrm{lab}}=11.8$ and $30.0 \mathrm{MeV}$ leading to the first excited $1 / 2_{1}^{+}, 3.09 \mathrm{MeV}$ state. The data points are taken from Refs. $[2,8,9]$. Right panel: CRC fit of the ${ }^{10} \mathrm{Be}\left(\mathrm{d}, \mathrm{p}_{1}\right)^{11} \mathrm{Be}$ reaction at $E_{\mathrm{lab}}=12$ and $25 \mathrm{MeV}$ leading to the first excited $1 / 2_{1}^{-}, 0.32 \mathrm{MeV}$ state of ${ }^{11} \mathrm{Be}$. The data points are taken from Refs. $[10,11]$. The lines correspond to the calculations with different overlap $n+{ }^{12} \mathrm{C}$ and $n+{ }^{10} \mathrm{Be}$ wave functions.

The ${ }^{11} \mathrm{Be}$ nucleus in the $1 / 2^{+}$g.s. is the most striking example of a nucleus with one-neutron halo [20, 21]. The $d+{ }^{10} \mathrm{Be}$ OPs at 12.0 and $25 \mathrm{MeV}$ incident deuteron energies are adopted form Refs. [10, 11]. Optical potentials describing the $p+{ }^{11} \mathrm{Be}$ interaction in the exit channel and the $p+{ }^{10} \mathrm{Be}$ interaction at half the incident deuteron energy are adopted from the global parameterizations [14,22] with slightly changed imaginary and spin-orbit parts, and Ref. [23]. The results of calculations are shown in Fig. 1 (right panel). The ANC values extracted from the $\left(d, p_{0}\right)$ reaction, $C^{\exp }{ }_{10 \mathrm{Be}-\mathrm{n}}\left(1 / 2^{+}\right)=0.723 \pm 0.016$ $\mathrm{fm}^{-1 / 2}$ at $E_{\mathrm{lab}}=12 \mathrm{MeV}$ and $0.715 \pm 0.035$ at $E_{\mathrm{lab}}=25 \mathrm{MeV}$ agree very well between each other. The average rms radius of the last neutron in the $1 / 2^{+}$g.s. of ${ }^{11} \mathrm{Be}$ is found to be very large, $R_{\mathrm{rms}}=$ $7.60 \pm 0.25 \mathrm{fm}$ and the relative weight of its asymptotic part reaches $96 \%$.

The ${ }^{10} \mathrm{Be}\left(\mathrm{d}, \mathrm{p}_{1}\right){ }^{11} \mathrm{Be}$ reaction at $E_{\mathrm{lab}}=12$ and $25 \mathrm{MeV}$ leading to the $1 / 2_{1}{ }^{-}$first excited state exhibits all features of a peripheral reaction, because the empirical ANCs do not change under moderate changes of the sp potential. We find the ANC values: $C^{\exp }{ }_{10 \mathrm{Be}-\mathrm{n}}\left(1 / 2^{-}\right)=0.133 \pm 0.004 \mathrm{fm}^{-1 / 2}$ at $E_{\mathrm{lab}}=12$ 
$\mathrm{MeV}$ and $0.128 \pm 0.006 \mathrm{fm}^{-1 / 2}$ at $E_{\mathrm{lab}}=25 \mathrm{MeV}$, which are in excellent agreement between each other. The stability of this state is kept up due to a centrifugal barrier formed by the orbital angular momentum $l=1$ of a neutron with respect to the core, despite the fact that this state lays only 0.184 $\mathrm{MeV}$ lower that the neutron-emission threshold. The asymptotic behaviour of the sp and Whittaker functions becomes the same at very long distances, about $40 \mathrm{fm}$, which requires a large integration range, up to $60 \mathrm{fm}$, for the correct accounting of the asymptotic behaviour of the wave functions. The sp overlap wave functions $u_{A n}(r)$ generated by the ${ }^{10} \mathrm{Be}-n$ interaction potential with enlarged geometrical parameters can correctly describe the shape of the angular distributions (some examples of calculations are shown in Fig. 1, right panel). The rms radius of the last neutron is found to be $4.58 \pm 0.25 \mathrm{fm}$, i.e. a factor of 1.66 smaller than that for the ground $1 / 2^{+}$state of ${ }^{11} \mathrm{Be}$, but approximately a factor of 1.4 greater than that of a normal nuclear state without halo. The asymptotic part of the rms radius contributes about $50 \%$. The probability of the last neutron to be outside the range of the interaction radius $R_{\mathrm{N}}=5 \mathrm{fm}$ is about $80 \%$ for sp overlap wave functions used in our calculations. Thus, we can associate the rms radius of the last neutron in the first excited $1 / 2^{-}$state of

${ }^{11} \mathrm{Be}$ with the halo radius, in accordance with criteria presented in Ref. [24].

To conclude, we have to stress that modern facilities for generation of exotic isotopes and their studies using inverse kinematics have caused a rebirth of interest to the deuteron-induced reactions, which open new possibilities to study halo structures in the excited states of exotic nuclei. The ANC approach can be one of alternative methods useful for estimating the radii of these short-lived states.

\section{References}

1. T. Otsuka, N. Fukunishi, and H. Sagawa, Phys. Rev. Lett. 70, 1385 (1993).

2. Z. H. Liu et al., Phis Rev C 64, 034312 (2001).

3. L. D. Blokhintsev, I. Borbely, and E. I. Dolinskii, Fiz. Elementar. Chastits Atom. Yadra 8, 1189 (1977) [ Sov. J. Part. Nucl. 8, 485 (1977)].

4. A. N. Danilov et al., Phys. Rev. C80, 054603 (2009).

5. A. A. Ogloblin, T. L. Belyaeva, A. S. Demyanova, and S. A. Goncharov, EPJA 60, (2013).

6. A. S. Demyanova et al., Int. J. Mod. Phys. E 20, 915 (2011).

7. A. A. Ogloblin et al., Phys. Rev. C 84, 054601 (2011).

8. R. Van Dantzig and L. A. Ch. Koerts, Nucl. Phys. 48, 177 (1963).

9. H. Ohnuma et al., Nucl. Phys. A448, 205 (1986).

10. D. L. Auton, Nucl. Phys. A157, 305 (1970).

11. B. Zwieglinski et al., Nucl. Phys. A315, 124 (1979).

12. G. R. Satchler, Direct Nuclear Reactions, Clarendon Press, Oxford, 1983.

13. I. J. Thompson, FRESCO user's manual and code, available from the author.

14. B. A. Watson, P. P Singh, and R. E. Segel, Phys. Rev. 182, 977 (1969).

15. P. Bem, O. Karban, and J. Nemec, Czech. J. Phys. B 18, 423 (1968).

16. G. R. Satchler, Nucl. Phys. 85, 273 (1966).

17. N. K. Timofeyuk and R. C. Johnson, Phys. Rev. Lett. 110, 112501 (2013).

18. S. A. Goncharov, I. R. Gulamov, E. A. Romanovsky, N. K. Timofeyuk, and K. V. Shitikova, J. Phys. G: Nucl. Part. Phys. 15, 1431 (1989).

19. S. A. Goncharov, J. Dobesh, E. I. Dolinsky, A.M. Mukhamedzhanov, and J. Cejpek, Yad. Fiz. 35, 662 (1982) [Sov. J. Nucl. Phys. 35, 383 (1982)].

20. P. G. Hansen, A. S. Jensen, and B. Jonson, Ann. Rev. Nucl. Part. Sci. 45, 591 (1995).

21. W. Nörtershäuser et al., Phys. Rev. Lett. 102, 062503 (2009).

22. F. D. Becchetti and G. W. Greenlees, Phys. Rev. 182, 1190 (1969).

23. N. K. Timofeyuk and R. C. Johnson, Phys. Rev. C59, 1545 (1999).

24. B. Jonson, Phys. Rep. 389, 1 (2004). 